IGCS20_1325

\section{OUTCOMES AFTER THE REGIONALIZATION OF CARE FOR HIGH RISK ENDOMETRIAL CANCERS: A POPULATION-BASED STUDY}

${ }^{1} \mathrm{~A}$ Nica* ${ }^{2}{ }^{2} \mathrm{R}$ Sutradhar, ${ }^{3} \mathrm{R}$ Kupets, ${ }^{3} \mathrm{~A}$ Covens, ${ }^{3} \mathrm{D}$ Vicus, ${ }^{2} \mathrm{Q}$ Li, ${ }^{4} \mathrm{~S}$ Ferguson. ${ }^{1}$ University of Toronto, Canada; ${ }^{2}$ CIES, Canada; ${ }^{3}$ Sunnybrook Health Sciences Centre, Canada; ${ }^{4}$ Princess Margaret Cancer Centre, Canada

\subsection{6/ijgc-2020-IGCS.263}

Objectives In June 2013, the agency responsible for advancing cancer care in Ontario, Canada, published practice guidelines recommending that gynaecologic oncologists (GOs) at designated centers manage the treatment of patients with high grade endometrial cancers. This study examines the effects of this regionalization of care on patient outcomes.

Methods In this retrospective cohort study, patients diagnosed with non-endometrioid high risk endometrial cancer (serous, carcinosarcoma, clear cell, undifferentiated) from 2003-2017 were identified using province-wide administrative databases.

Results We identified 3518 patients with high risk endometrial cancer. The case mix as represented by patient comorbidities and disease stage distribution did not differ significantly between the two regionalization periods. There was a significant increase $(69 \%$ to $85 \%, \mathrm{p}<0.001)$ in the proportion of primary surgeries performed by GOs after regionalization, which was not explained by secular trends. After regionalization, the proportion of patients who had surgical staging $(50 \%$ to $63 \%, \mathrm{p}<0.001)$, and the proportion of patients who received adjuvant treatment $(65 \%$ to $71 \%, \mathrm{p}<0.001)$ increased significantly. After adjusting for age, stage, and comorbidities, there was an increase in overall survival (HR 0.85 (0.73$0.99), p=0.04$ ) after regionalization.

Conclusions The publication of a regionalization policy for the treatment of high risk histology endometrial cancers in Ontario led to an increase in the proportion of surgeries performed by GOs, surgical staging and adjuvant treatment. This also translated into a significant improvement in patient survival.

\section{IGCS20_1326}

\section{PRIMITIVE CARCINOMA OF THE FALLOPIAN TUBE: CLINICAL AND PROGNOSTIC FEATURES}

${ }^{1} \mathrm{O}$ Kaabia*, ${ }^{1} \mathrm{O}$ Ben Ahmed, ${ }^{2} \mathrm{~T}$ Yaacoubi, ${ }^{2} \mathrm{M}$ Mokni, ${ }^{1} \mathrm{M}$ Bibi, ${ }^{1} \mathrm{H}$ Khairi. ${ }^{1}$ Université de Sousse, Faculté de Médecine de Sousse, Hôpital Farhat Hached, LR12ESO3, 4000, Sousse, Tunisie; Tunisia; ' Université de Sousse, Faculté de Médecine de Sousse, Hôpital Farhat Hached, Department of Pathology, 4000, Sousse, Tunisie;, Tunisia

\subsection{6/ijgc-2020-IGCS.264}

Objectives Primitive Carcinoma of the Fallopian tube is extremely rare and represents 0,1 to $1 \%$ of all malignant tumors of the pelvis. The aim of this study is to describe its clinical and prognostic features.

Methods It is a retrospective study conducted in the Tunisian Central Cancer Registry during a 15 year period (2004 2018) collecting all the pathologically established and confirmed cases of primitive carcinoma of the Fallopian tube.
Results The incidence of Primitive Carcinoma of the Fallopian tube was $1 / 1559$ of all the Ovarian, fallopian tube, and peritoneal cancers in our registry. The mean age at the diagnosis was 54.2 years. Pelvic pain was the main symptom. Pelvic clinical examination reported a mass in $86 \%$ of the cases. Pelvic ultrasound revealed a para-uterine image in all cases but could not differentiate between an ovarian tumor, a Fallopian tube one, or a tubo-ovarian infectious abscess. The perioperative diagnosis was evocated during laparoscopy in only $12 \%$ of the cases. The different pathological diagnoses of Primitive Carcinoma of the Fallopian tube were: tubal carcinoma in situ, tubal Carcinoma, tubal adenocarcinoma, and tubal papillar adenocarcinoma. An optimal cytoreduction surgery was possible in $86 \%$ of the cases. During the follow-up, the recurrence rate was $24 \%$ and the overall survival rate at 3 years was $82 \%$.

Conclusions Primitive Carcinoma of the Fallopian tube is a very rare entity in our daily practice. Diagnosis is rarely made before surgery or the pathological study. The 3-year prognosis is relatively good.

\section{IGCS20 1328}

\section{NEO-ADJUVANT CHEMOTHERAPY FOR CERVICAL CANCER DURING PREGNANCY: A RETROSPECTIVE CASE SERIES}

${ }^{1} \mathrm{~A}$ Lopez ${ }^{*},{ }^{2} \mathrm{~J}$ Rodríguez, ${ }^{3} \mathrm{E}$ Estrada, ${ }^{1} \mathrm{C}$ Chavez, ${ }^{4} \mathrm{C}$ Amaro, ${ }^{5} \mathrm{~A}$ Aragona, ${ }^{6} \mathrm{C}$ De Padua, ${ }^{7} \mathrm{~A}$ Borges Garnica, ${ }^{8} \mathrm{G}$ Rendón, ${ }^{9} \mathrm{~A}$ Almeciga, ${ }^{10} \mathrm{O}$ Serrano, ${ }^{11} \mathrm{~S}$ Scasso, ${ }^{11} \mathrm{~J}$ Laufer, ${ }^{11} \mathrm{D}$ Greif, ${ }^{12} \mathrm{~F}$ Taranto, ${ }^{13} \mathrm{~A}$ Mora, ${ }^{14} \mathrm{R}$ Pareja. ${ }^{1}$ Department of Gynecologic surgery. Instituto Nacional de Enfermedades Neoplásicas, Peru; ${ }^{2}$ Department of Gynecology Oncology. Instituto Nacional de Cancerología. Department of Gynecology and obstetrics. Section of Gynecology Oncology. Fundación Santa Fe de Bogotá, Colombia; ${ }^{3}$ Hospital General San Juan de Dios, Guatemala; ${ }^{4}$ Hospital Cayetano Heredia, Peru; ${ }^{5}$ Hospital Municipal de Oncología Marie Curie, Argentina; ${ }^{6}$ Hospital de Câncer de Barretos - Fundação Pio XII, Brazil; ${ }^{7}$ Unidad de Terapia Antineoplásica (UTAN).Centro Médico Guerra Méndez, Venezuela; ${ }^{8}$ Department of Gynecologic Oncology. Instituto de Cancerología- Las Américas-Auna. Hospital General, Colombia; ${ }^{9}$ Department of Gynecology Oncology. Instituto Nacional de Cancerología, Colombia; ${ }^{10}$ Department of Gynecologic Oncology. Hospital Militar Central de Bogotá, Colombia; ${ }^{11}$ Department of Gynecologic Oncology. Hospital Pereira Rossell, Uruguay; ${ }^{12}$ Department of Gynecology. Hospital de Clínicas Dr. Manuel Quintela. Universidad de la República, Uruguay; ${ }^{13}$ Department of Gynecology Oncology. Hospital México, Costa Rica; ${ }^{14}$ Clínica de Oncología Astorga. Universidad Pontificia Bolivariana. Department of Gynecology Oncology. Instituto Nacional de Cancerología, Colombia

\subsection{6/ijgc-2020-IGCS.265}

Introduction Neo-adjuvant chemotherapy (NACT) in cervical cancer during pregnancy may help in disease control while fetal maturity is reached, before providing a definitive oncological treatment. The objective of this study is to describe obstetric and oncological outcomes in patients diagnosed with cervical cancer stage IB1-IVA (FIGO 2009) during pregnancy, who received this treatment approach.

Methods A multicenter retrospective review was conducted in 12 institutions from 7 Latin-American countries, between January 2007 and December 2018. Data collected included clinical characteristics, NACT agents, definitive treatment, obstetric and oncologic outcomes.

Results Twenty-nine patients were included. Mean age was 33.8 years $(+5.2)$. Twelve $(41.4 \%)$ women were diagnosed at early stage, and 17 (58.6\%) in locally advanced stage. Carboplatin/Paclitaxel was the most frequent combination used (55.2\%). Median number of cycles was 3 (1-6). Median 